\title{
Asociación del estado nutricional y funcional en ancianos con fractura de cadera
}

\author{
Association of nutritional and functional status in \\ the elderly with hip fracture
}

\author{
Sandra Milena Caicedo-Correa, Samir Alejandro Aruachan-Torres, \\ Ana Isabel Segura-Valencia, Diego Andrés Chavarro-Carvajal \\ - Bogotá, D.C. (Colombia)
}

\section{Resumen}

Introducción: las fracturas de cadera son una complicación frecuente y grave en las personas ancianas. Existen múltiples factores que se asocian con las fracturas de caderas en los ancianos, entre los que se destacan el estado nutricional y la funcionalidad de los afectados. El estado nutricional está condicionado en parte por el proceso de envejecimiento y por los cambios físicos, mentales, sociales y ambientales que se asocian a éste. La desnutrición es un problema de gran relevancia en la población anciana y que puede influir en el pronóstico asociado a diversos procesos patológicos. La presencia de alteraciones nutricionales se han relacionado directamente con el estado funcional.

Objetivo: determinar el estado funcional y nutricional previo, y su asociación en ancianos con fractura de cadera.

Material y métodos: se estudiaron 177 ancianos con fractura de cadera del $1^{\circ}$ enero de 2013 hasta el 31 de diciembre de 2016 que ingresaron al programa de ortogeriatría del Hospital Universitario San Ignacio en Bogotá, Colombia. A todos los pacientes se les analizó el estado funcional y nutricional dos semanas previo al evento. Se utilizó estadística descriptiva y análisis inferencial para pruebas de asociación con x2.

Resultados: el $45.76 \%$ eran independientes para sus actividades básicas de la vida diaria y $54.23 \%$ presentaban algún grado de deterioro funcional. El $26.55 \%$ presentaban malnutrición, el $45.76 \%$ riesgo de malnutrición y $27.68 \%$ un estado nutricional adecuado. De los pacientes con algún grado de malnutrición (MNA<24), solo $32.81 \%$ eran independientes funcionalmente y $79.59 \%$ con adecuado estado nutricional eran independientes. La asociación entre malnutrición y dependencia funcional se estimó con un $\mathrm{RP}=3.94$ (2.09-7.43 95\% IC) con $\mathrm{p}=0.00$.

Conclusión: el estado nutricional y funcional son factores asociados a presentar fractura de cadera. (Acta Med Colomb 2019; 44: 7-10).

Palabras clave: fractura de cadera, estado nutricional, funcionalidad, anciano.

\footnotetext{
Abstract

Introduction: hip fractures are a frequent and serious complication in elderly people. There are multiple factors that are associated with hip fractures in the elderly, among which are the nutritional status and functionality of those affected. The nutritional status is partly conditioned by the aging process and the physical, mental, social and environmental changes associated with it. In the elderly population malnutrition is a problem of great relevance and can influence the prognosis associated with various pathological processes. The presence of nutritional alterations has been directly related to the functional state.

Objective: to determine the previous functional and nutritional status, and its association with hip fracture in elderly people.

Materials and methods: from January 1, 2013 to December 31, 2016, 177 elderly patients with hip fracture who entered the orthogeriatric program of the San Ignacio University Hospital in Bogotá, Colombia, were studied. All patients were analyzed for functional and nutritional status two weeks prior to the event. Descriptive statistics and inferential analysis were used for association tests with $\mathrm{x} 2$.
}

Dra. Sandra Milena Caicedo Correa: Geriatra. Unidad de Geriatría Hospital Universitario San Ignacio, Instituto de Envejecimiento, Pontificia Universidad Javeriana; Doctores: Samir Alejandro Aruachan y Ana Isabel Segura Valencia: Residentes de Especialización en Geriatria, Pontificia Universidad Javeriana, Hospital Universitario San Ignacio; Dr. Diego Andrés Chavarro-Carvajal: Internista, Geriatra, Epidemiólogo, Unidad de Geriatría, Hospital Universitario San Ignacio. Instituto de Envejecimiento, Pontificia Universidad Javeriana. Bogotá, D.C. (Colombia). Correspondencia. Dra. Sandra Milena CaicedoCorrea. Bogotá, D.C. (Colombia). E-mail: sandycaicedo07@gmail.com Recibido: 24/V/2017 Aceptado: 30/I/2019 
Results: $45.76 \%$ were independent for their basic daily life activities, and $54.23 \%$ had some degree of functional deterioration. $26.55 \%$ had malnutrition, $45.76 \%$ risk of malnutrition and $27.68 \%$ an adequate nutritional status. Of the patients with some degree of malnutrition (MNA <24), only $32.81 \%$ were functionally independent and $79.59 \%$ with adequate nutritional status were independent. The association between malnutrition and functional dependence was estimated with a PR = $3.94(2.09-7.4395 \% \mathrm{CI})$ with $\mathrm{p}=0.00$.

Conclusion: nutritional and functional status are factors associated with hip fracture presentation. (Acta Med Colomb 2019; 44: 7-10).

Key words: hip fracture, nutritional status, functionality, elderly.

\section{Introducción}

Las fracturas de cadera son una complicación frecuente y grave en las personas ancianas. Existen múltiples factores asociados con las fracturas de cadera en los ancianos, entre los que se destacan el estado nutricional y la funcionalidad de los afectados (1). Sin embargo, el estado nutricional ha despertado gran interés en la recuperación pre y post operatoria en términos de funcionalidad y recuperación de autonomía (2). El estado nutricional está condicionado en parte por el proceso de envejecimiento y por los cambios físicos, mentales, sociales y ambientales que se asocian a éste (3).

La desnutrición es un problema de gran relevancia en la población anciana y que puede influir en el pronóstico asociado a diversos procesos patológicos.

Los estados de malnutrición son frecuentes en gran parte de los pacientes fracturados, en estudios previos se manifiesta que $60 \%$ de los pacientes se encontraban desnutridos al ingreso (4) o desarrollarán déficits nutricionales graves durante su hospitalización (5).

Los pacientes con fractura de cadera por lo general tienen varias comorbilidades: enfermedad crónica, depresión, deterioro cognitivo, sarcopenia, desnutrición y alteración de la movilidad, fuerza y visión (6). Estas comorbilidades agravan el problema de la desnutrición, debido a ingesta deficiente, deterioro físico y cognitivo, depresión, soledad y pobreza, enfermedades agudas y crónicas y la toma de múltiples medicamentos (7). Así, la desnutrición puede influir en la recuperación funcional después de la fractura de cadera, y la intervención nutricional en fase temprana puede mejorar los resultados de los pacientes con fractura de cadera (8).

La valoración funcional es uno de los pilares de la valoración geriátrica integral (VGI) y consiste en el estudio de las capacidades físicas del anciano en todo su espectro, desde las tareas motoras más simples y su capacidad de autocuidado hasta el ejercicio y la independencia en el medio ambiente.

Muchos pacientes con fractura de cadera sufren de pérdida de independencia funcional, y $60 \%$ de ellos no recuperan la capacidad funcional previa (7). El 10-20\% de los que viven en casa antes de la fractura de cadera será dado de alta a la institucionalización, y sólo un tercio recuperará el estado funcional anterior tres meses después de la fractura de cadera (8). La presencia de alteraciones nutricionales se ha relacionado directamente con el estado funcional, por lo tanto la fuerza muscular como la nutrición deben abordarse en el tratamiento integral de la fractura de cadera y en la rehabilitación (9).

El objetivo de este estudio fue determinar el estado funcional y nutricional previo, y su asociación en ancianos con fractura de cadera.

\section{Material y métodos}

La información fue tomada de la base de datos del protocolo de ortogeriatría del Hospital Universitario San Ignacio, del $1^{\circ}$ de enero de 2013 al 31 de diciembre de 2016.

Se realizó un estudio descriptivo de corte analítico. Esta base de datos incluía informes como edad, género, funcionalidad, cognición, estado nutricional, mes de la caída, situación social, mecanismo de caída y sitio de caída, tipo de fractura, sitio de la fractura, fármacos más frecuentemente tomados y patologías asociadas.

El protocolo de investigación fue aprobado por el comité de ética en investigación de la Pontificia Universidad Javeriana y Hospital Universitario San Ignacio.

Inicialmente se incluyeron 196 pacientes que presentaron fractura de cadera en el HUSI en ese periodo de tiempo, de los cuales se excluyeron cinco pacientes por fractura patológica, y 14 pacientes por otras causas (remisión, edad menor de 65 años, no valorados por servicio de geriatría, no candidatos a manejo quirúrgico), para un total de 177 pacientes incluidos en el estudio.

En este estudio se tomó como variable dependiente, un mal estado nutricional y como variables independientes, el estado funcional dos semanas previas al ingreso, la edad y el sexo (ser mujer) en ancianos con fractura de cadera. La funcionalidad fue evaluada mediante la aplicación de la escala de Barthel dos semanas previo al ingreso del programa (10), clasificando los pacientes en independientes (100-95), dependencia leve (90-60), dependencia moderada (55-40), dependencia severa/grave (35-20) y dependencia total $(<20)(16)$. El estado nutricional fue evaluado mediante la aplicación del Mininutritional Assessment (MNA), clasificando los pacientes con estado nutricional normal (24-30 puntos), riesgo de malnutrición (17-23.5) y malnutrición $(<17)(17-19))$.

Los datos fueron analizados mediante modelos bivariados para determinar la asociación entre variables dependientes 
(malnutrición) e independientes (dependencia funcional, edad y ser mujer).

Se realizó un modelo de regresión de Poisson ajustado para obtener una razón de prevalencia (RP), con intervalo de confianza de $95 \%$. Se estableció el nivel de significancia estadística en $\mathrm{p}<0.05$. Los datos fueron analizados con el programa STATA para iOS.

\section{Resultados}

En el estudio se incluyeron 177 pacientes adultos mayores, de los cuales $31.07 \%$ pertenecían al sexo masculino y

Tabla 1. Descripción de la población.

\begin{tabular}{|c|c|}
\hline \multicolumn{2}{|c|}{ Descripción sociodemográfica (n=177) } \\
\hline Variable & Promedio (DE) ó n (\%) \\
\hline Edad & $81.52(7.23)$ \\
\hline \multicolumn{2}{|l|}{ Sexo } \\
\hline Hombre & $55(31.07)$ \\
\hline Mujer & $122(68.93)$ \\
\hline \multicolumn{2}{|l|}{ Barthel } \\
\hline 100-95 (independiente) & $81(45.76)$ \\
\hline 90-60 (dependencia leve) & $46(25.99)$ \\
\hline 55-40 (dependencia moderada) & $26(14.69)$ \\
\hline 35-20 (dependencia grave) & $19(10.73)$ \\
\hline$<20$ (dependencia total) & $5(2.82)$ \\
\hline \multicolumn{2}{|l|}{ Trastorno neurocognoscitivo } \\
\hline Sin TNC & $76(42.94)$ \\
\hline DCL & $10(5.65)$ \\
\hline TNC leve & $31(17.51)$ \\
\hline TNC moderado & $49(27.68)$ \\
\hline TNC severo & $11(6.21)$ \\
\hline \multicolumn{2}{|l|}{ Estado nutricional } \\
\hline Adecuado & $49(27.68)$ \\
\hline Riesgo MN & $81(45.76)$ \\
\hline Malnutrición & $47(26.55)$ \\
\hline \multicolumn{2}{|l|}{ Tipo de fractura } \\
\hline Intertrocantérica & $104(58.76)$ \\
\hline Transcervical & $58(32.77)$ \\
\hline Subtrocantérica & $15(8.47)$ \\
\hline \multicolumn{2}{|l|}{ Comorbilidad } \\
\hline 0 & $9(5.08)$ \\
\hline 1 & $21(11.86)$ \\
\hline 2 & $43(24.29)$ \\
\hline 3 & $44(24.86)$ \\
\hline $40>$ & $60(33.90)$ \\
\hline Polifarmacia & $95(53.67)$ \\
\hline
\end{tabular}

$68.93 \%$ al sexo femenino. La edad promedio de 81.52 años. Del total de pacientes, $45.76 \%$ eran independientes para las actividades básicas de la vida diaria y $54.24 \%$ tenían algún grado de dependencia funcional dos semanas previo al ingreso. A nivel nutricional, sólo $27.68 \%$ de los pacientes tenían un adecuado estado nutricional, el resto presentaban algún grado de malnutrición (Tabla 1).

En el análisis bivariado de los pacientes con algún grado de malnutrición $(\mathrm{MNA}<24)$, solo $32.81 \%$ eran independientes funcionalmente, y $79.59 \%$ con adecuado estado nutricional eran independientes. Así mismo, de los pacientes con algún grado de malnutrición, $67.19 \%$ tenían algún compromiso funcional, mientras que solo $20.41 \%$ de los pacientes con adecuado estado nutricional tenían algún grado de compromiso funcional.

Utilizando el análisis de regresión de Poisson, se determinó una asociación positiva entre estado nutricional y dependencia funcional con una razón de prevalencia $(\mathrm{RP}) \mathrm{de}$ 4.62 (IC 95\% 2.46-8.68; p=0.00). Igualmente, tras realizar el análisis de regresión logística multivariado ajustado por las variables de confusión (edad, sexo) la asociación entre malnutrición y dependencia funcional fue significativa con una RP de 3.94 (IC 95\% 2.09-7.43; p=0.00) (Tablas 2 y 3).

\section{Discusión}

Las fracturas de cadera en el anciano representan hoy día un problema de salud pública importante, debido a la prevalencia de estas en la población anciana (11).

Con el análisis de los datos se evidenció una asociación independiente entre fractura de cadera y pacientes con algún grado de malnutrición y alteración funcional. Se encontró que $54.24 \%$ de los pacientes con fractura de cadera tenían algún grado de deterioro funcional. Así mismo el 72.3\% de los pacientes tenían algún grado de malnutrición, cifra superior a los estudios mencionados anteriormente.

Como se ha descrito la fractura de cadera conlleva una importante carga de enfermedad, asociándose a discapacidad física, disminución en la calidad de vida, muerte e incremento en la utilización de servicios de salud (12). Adicionalmente la presencia de algún grado de malnutrición se ha asociado a mayor alteración cognitiva, mayor dependencia funcional y es un factor independiente para morbilidad y mortalidad, mayor estancia hospitalaria, severidad de complicaciones agudas y limitación para la rehabilitación (13). El estado nutricional prefractura fue factor predictor para estado funcional al momento del alta independiente de otras comorbilidades, fuera de esto existe una asociación directa con peores tasas de recuperación (8).

Tomando en cuenta lo anterior, debe considerarse el abordaje médico integral, el cual además de generar mejoramiento global de la carga de enfermedad y disminuye estancia hospitalaria al permitir rehabilitación temprana y uso de suplementación nutricional (14). Finalmente, debido a que múltiples estudios han coincidido en que la malnutrición es un adecuado predictor para un peor estado funcional 
Tabla 2. Análisis bivariado estado nutricional (resultado Mininutritional Assessment) y dependencia funcional (resultado escala Barthel).

\begin{tabular}{|c|c|c|c|c|c|c|}
\hline $\begin{array}{c}\text { Estado nutricional/ } \\
\text { Dependencia funcional }\end{array}$ & $\begin{array}{c}\text { Independiente } \\
\quad(100-95)\end{array}$ & $\begin{array}{c}\text { Dependencia leve } \\
(90-60)\end{array}$ & $\begin{array}{c}\text { Dependencia } \\
\text { moderada }(55-40)\end{array}$ & $\begin{array}{c}\text { Dependencia grave } \\
(35-20)\end{array}$ & $\begin{array}{c}\text { Dependencia total } \\
(<20)\end{array}$ & Total \\
\hline Adecuado & $39(79.59)$ & $6(12.24)$ & $4(8.16)$ & $0(0)$ & $0(0)$ & $49(100)$ \\
\hline Riesgo malnutrición & $29(35.80)$ & $32(39.51)$ & $11(13.58)$ & $9(11.11)$ & $0(0)$ & $81(100)$ \\
\hline Malnutrición & $13(27.66)$ & $8(17.02)$ & $11(23.4)$ & $10(21.28)$ & $5(10.64)$ & $47(100)$ \\
\hline Total & $81(45.76$ & $46(25.99)$ & $26(14.69)$ & $19(10.73)$ & $5(2.82)$ & $177(100)$ \\
\hline
\end{tabular}

Tabla 3. Asociación entre estado nutricional y funcional.

\begin{tabular}{|l|c|c|}
\hline \multicolumn{1}{|c|}{ Estado nutricional $\backslash$ Estado funcional } & $>90$ (independiente ABVD) & <90 (dependencia ABVD) \\
\hline$<24$ (Malnutrición) & $42(32.81)$ & $86(67.19)$ \\
\hline$>24$ (Estado nutricional satisfactorio) & $39(79,59)$ & $10(20.41)$ \\
\hline Total & 81() & $(100)$ \\
\hline ABVD: actividades básicas de la vida diaria. $\quad$ X2 31.24 $\mathrm{p}=0.000 \quad$ RP crudo 4.62 (IC 2.46-8.68) p (0.00) & RP ajustado por edad y ser mujer: 3.94 (IC 2.09-7.43) p=0.00 \\
\hline
\end{tabular}

posterior a la fractura de cadera se considera la implementación de suplementos nutricionales y rehabilitación temprana dentro del manejo de adultos mayores diagnosticados con esta enfermedad (15).

Este estudio tiene algunas limitaciones, ya que al tener un diseño de corte transversal, no es posible determinar causalidad, por lo que únicamente se puede reportar asociación entre las variables estudiadas. Sin embargo, también se deben identificar algunas fortalezas, ya que a nivel tanto nutricional como funcional se logró clasificar con certeza a los pacientes en cada grupo utilizando las herramientas de escala de Barthel y MNA respectivamente.

Estudios posteriores deben centrarse en la población adulta mayor de nuestro medio, para determinar el impacto de nuevas estrategias dirigidas a rehabilitación e intervención nutricional perioperatoria dentro de un enfoque integral que permita al paciente recuperar funcionalidad, prevenir desenlaces fatales y conservar estado cognitivo posterior a evento catastrófico como lo es la fractura de cadera.

\section{Referencias}

1. González-Montalvo JI, Gotor P, Martín-Vega A, Alarcón T, Mauleón J, Gil E, García E, Alonso J. La unidad de ortogeriatría de agudos. Evaluación de su efecto en el curso clínico de los pacientes con fractura de cadera y estimación del impacto económico. Rev Esp Geriatr Gerontol 2011;46(4):193-9.

2. Neira M, López-Dóriga P, Thuissard I, Sanz-Rosa D, Arias E, Bielza R, Gomez J. Grip strength and functional recovery after hip fracture: An observational study in elderly population. European Geriatric Medicine . 2016;768:1-5 (http://dx.doi. org/10.1016/j.eurger.2016.05.002).

3. Miralles R, Conejo A, López E. Prevención de la fractura de cadera en ancianos. En Avellana J, Ferrández L. Guía de buena práctica clínica en Geriatría. Anciano Afecto de fractura de cadera. 1 ed. España: Elsevier ;2007. P. 85-104

4. Shyu YI, Chen MC, Liang J, Wu CC, Su JY. Predictors of functional recovery for hip fractured elders during 12 months following hospital discharge: a prospective study on a Taiwanese sample. Osteoporos Int. 2004;15:475-82.

5. Koren-Hakim T, Weiss A, Hershkovitz A, Otzrateni I, Grosman B, Frishman S, Beloosesky Y. The relationship between nutritional status of hip fracture operated elderly patients and their functioning, comorbidity and outcome. Clin Nutr 2012; 31(6): 917-921.
6. Wang HP, Lian J, Kuo LM, Chen CY, Shyu YI.Trajectories of nutritional status and cognitive impairment among older taiwanese with hip fracture. J Nutr health Aging.2017;21(1):38-45

7. Tal S, Gurevich A, Sagiv S, Guller V.Predictors of mortality in hip fracture patients. European Geriatric Medicine.2016;6(7):561-565

8. Inoue T, Misu S, Tanaka T, Sakamoto H, Iwata K, Chuman Y, Ono R. Prefracture nutritional status is predictive of functional status at discharge during the acute phase with hip fracture patients: A multicenter prospective cohort study. Clinical Nutrition. 2017;36(5):1320-1325.

9. Goisser S, Schrader E, Singler K, Bertsch T, Gefeller O, Biber R, Bail HJ, Sieber CC, Volkert D. Malnutrition according to mini nutritional assessment is associated with severe functional impairment in geriatric patients before and up to 6 months after hip fracture. J Am Med Dir Assoc 2015;16(8):661-667.

10. Mahoney, F., Barthel, D. Functional evaluations: The Barthel index. Maryland State Medical Journal, 1965, 14: 61-65.

11. Bardales Y, González-Montalvo JI, Abizanda P,Alarcon M. Guías clínicas de fractura de cadera. Comparación de sus principales recomendaciones. Rev Esp Geriatr Gerontol 2012;47(5):220-227.

12. Koot V, Peeters P, Jong J, Clevers G, Van der Werken C. Functional results after treatment of hip fracture: a multi- centre, prospective study in 215 patients. Eur J Surg 2003;166(6):480-485.

13. Wyers C, Reijven P, Evers S, Willems P, Heyligers I, Verburg A, Van Helden S, Dagnelie P. Cost-effectiveness of nutritional intervention in elderly subjects after hip fracture. A randomized controlled trial. Osteoporois Int 2013;24(1):151-162.

14. Alarcón T, Gonzalez J. Atención integral al anciano con fractura de cadera.. En Abizanda P, Rodriguez-Mañas L, Baztán J. Tratado de Medicina geriátrica. 1 ed. España:Eslsevier 2015. Cap 80. P.635-644.

15. Drevet $S$, Bioteau $C$, Mazière $S$, Couturier $P$, Merloz $P$, Tonetti J, Gavazzi G. Prevalence of protein-energy malnutrition in hospital patients over 75 years of age admitted for hip fracture. Orthop Traumatol Surg Res. 2014;100(6);669-674

16. Muñoz C, Rojas P, Nasri G. Valoración del estado funcional de adultos mayores con dependencia moderada y severa pertenecientes a un centro de salud familiar. Fisioter Pesq. 2015;22(1):76-83

17. Guigoz Vellas B, Garry P. Mininutritional Assessment:a practical assessment tool for grading the nutritional state of elderly patients. Facts Res Gerontol.1994;(supp12):15-60.

18. Guigoz Vellas B, Garry P. Assessing the nutritional status of the elderly: the Mini Nutritional Assessment s part of the geriatric evaluation. Nutr Rev.1996;54:S59-65.

19. Otero Mildred Rosero, Rosas Estrada Giovana Marcela. Valoración nutricional de las personas mayores de 60 años de la ciudad de pasto, colombia. Cienc. enferm. (Internet). 2017 Dic (citado 2018 Nov 06) ; 23( 3 ): 23-34. Disponible en: https://scielo.conicyt.cl/scielo.php?script=sci_ arttext\&pid=S0717-95532017000300023\&lng=es. http://dx.doi.org/10.4067/ S0717-95532017000300023. 